\title{
Neuropsychobiology 1985;13:215-216
}

\section{Subject Index}

Activation 141 Adoptive parents $7 \alpha$-Adrenoceptors $206<$ X2-Adrenoceptors 23 Affective disorders 17 Age psychosis 167 Aggression 1, 101, 194 Aggressive conduct disorder 7 Amino acids 160 Amitriptyline 31 AMP system 117 Animal models 55 Anticonvulsants 206 Antidepressant drugs 55 Antiepileptic drugs 201 Anxiety 187 Anxiogenic drugs 55 Anxiolytic drugs 55 Anxiolytics 194 Arousal 74 Atenolol 187 Attention 74, 136

Biologic parents 7

Biorhythms 17

B-Blockade and anxiety 187

Brain regions 26

Bromocriptine mesylate (Parlodel $\mathbb{\circledR}) 173$

Calcium antagonist 152

Carbon monoxide 136
Cardiac arrest, dog 147

- neurosis 12

Cerebral ischemia 152

Chronic alcoholism 97

Orcadian rhythm 111

Clonidine 23

Clouded consciousness 106

Cognitive functions 81

Comprehensive Psychopathological Rating

Scale 117, 180 Contingent negative variation 136 Coping 129

Correlation, canonical 117 Cortisol 157 Cybernetic 17 
Death risk 167 Depression 31, 111 Desipramine 160 Drug-refractory depression 111

EEG cartography 69, 141 Endogenous event-related potentials 81 Endogenous/reactive depression 160 Epidemiology 167 Epilepsy 206 Epileptic children 93 - patients 201 Epinephrine 157 Evoked potentials 74 , 136

Factor structure 180 Flunarizine 147 Flupentixol 81

Geriatric hospital, use patterns of

psychotropic drugs 38 German and Swedish version 180 Gerontopsychopharmacology 44

Human information processing 63 Hyperprolactinemia, neuroleptic-induced 173

Iconic recognition thresholds 63

Imipramine 160

Information processing 74,136

Learned immobility test 26 Lundby|Study 167

Man 23

Microcomputer 69 Milenperone 1,101 Minicomputer 69 Mortality 167

Neuroleptics 206 Neurological deficit 147 Neurophysiology 44 Nicotine 74, 136 Nomifensine 26 
Olfactory bulbectomy 26 Oligophrenic patients 101 ORG 2766, ACTH 4-9 analogue 63 Organic brain syndrome 44 Over-mortality 167 Oxiracetam 44

Paranoid reactive psychoses 106 Parasuicide 157 Paroxetine 31 Performance 74, 136 Period-amplitude EEG analysis 147 Permutation Fisher test 141 Persecutory affective psychoses 106 Petit mal 206 PharmacoEEG 152 Pharmacopsychological effects 81 Phenothiazines 173 Phobic behaviour 187 Placebo control 44 Plasma catecholamines 12 Power spectral EEG analysis 147 Premorbid personality 106 Prevalence 167 Propranolol 187 Psychogeriatric patients 1 Psychometry 44 Psychopathology 44 Psychotropic drugs 38

Quantitative EEG 44, 69

Rat 23, 194,206 Rating scales, psychiatric 117 ß-Receptor blockers 187 Reliability study 69 REM sleep 97

Scale construction 180 Serotonin metabolism 26 Sleep 23

- deprivation 111,201

- efficiency 97 
Sleep-wakefulness 201

Slow-wave sleep 97

Sobriety 97

Social interaction 55, 194

Somnopolygraphy 97

Somnopolygraphy 97

Spectral analysis 93
Spikes and waves 206

Spontaneous EEG paroxysms 206

Statistics 141 
Stress 129 Stressful event 129 Stress-related diseases 129 Survivorship 167

Tachistoscope 63

Thioridazine hydrochloride (Mellaril $\AA$ )

173 Tobacco 74, 136 
Total sleep time 97 Trazodone 26

Valproate therapy 93 Vigilance 44 Visual attention 141 - EEG analysis 147

Wakefulness 23 